\title{
Extracellular caspase-1 regulates hair follicle stem cell migration during wound- healing.
}

Subhasri Ghosh ${ }^{1}$, Akhil SHP Ananthan ${ }^{1}$, Sunny Kataria ${ }^{1}$, Neha Pincha ${ }^{1}$, Abhik Dutta ${ }^{1,2}$, Srilekha Athreya ${ }^{1}$, Aishwarya Bhosale ${ }^{1}$, Rakesh Dey ${ }^{1}$ and Colin Jamora ${ }^{1}$.

Affiliations:

1. IFOM - inStem Joint Research Laboratory, Institute for Stem Cell Biology and Regenerative Medicine, Bangalore, Karnataka, India

2. SASTRA Deemed University, Thanjavur, India.

Correspondence to be addressed to colinj@instem.res.in

Abstract: Migration of stem cells from one niche to another is a fundamental behavior observed during tissue morphogenesis, homeostasis, and repair ${ }^{1}$. A common thread running throughout these phenomena is the ability of stem cells to sense their environmental cues that, in turn, regulate their spatiotemporal localization with amazing precision. Perturbations of such cellular responses underlie a spectrum of pathologies ranging from developmental defects, tumor metastasis and ineffective wound closure ${ }^{2,3}$. In somatic tissues, the wound-healing process is a paradigm of the directed migration of various stem cell pools to the site of injury where they differentiate to replenish lost or damaged cells. While there has been substantial investment and progress in understanding the lineage trajectory of stem cells once they reach their destination, comparatively little is understood regarding the mechanisms guiding their chemotactic journey to the wound site. In the context of the skin, it has been shown nearly two decades ago that wounding activates various local epithelial stem cell pools, including multipotent hair follicle stem cells, to infiltrate the epidermis where they participate in the reconstruction of the damaged tissue ${ }^{4,5}$. However, elucidation of the environmental cues that coax these cells out of their hair follicle niche to the damaged epidermis has proven to be an intractable problem to solve. Using both an excisional wound and genetic mouse models of wound healing, we discovered that wounded keratinocytes secrete the enzyme Caspase-1. This protein is classically known as a critical component of the cytosolic macromolecular complex called an inflammasome that mediates the unconventional secretion of various cytokines including IL-1a 6 . Surprisingly, we find that the released caspase- 1 itself has a non-canonical role in the extracellular milieu. Through the Caspase Activation Recruitment Domain (CARD) of caspase-1, this protein is sufficient to initiate chemotaxis of hair follicle stem cells into the epidermis. The secretion of caspase- 1 has also been documented in many other pathological scenarios ${ }^{7,8}$ and we observed that the migration of HFSCs into the epidermis following UV irradiation of the skin is also caspase-1 dependent. Uncovering this novel function of Caspase- 1 facilitates a deeper understanding of the mechanistic basis of the epithelial hyperplasia found to accompany numerous inflammatory skin diseases.

Migration of hair follicle stem cells (HFSCs) into the epidermis is recapitulated in 


\section{a genetic model of wound healing}

A major hurdle in dissecting the mechanisms regulating the homing of HFSCs to the wound bed has been the limited number of responsive HFSCs that are restricted to the immediate proximity of the wound ${ }^{9}$. A genetic model of wound-healing that elicits a repair process throughout the skin in the absence of an injury would bypass this technical obstacle by amplifying the extensive intercellular crosstalk that takes place during the tissue regeneration program. Thus, we evaluated the potential of studying the mechanisms guiding HFSC migration in the mouse with the conditional knock-out of epidermal caspase-8 (C8cKO), which we previously reported to mimic many phenomena associated with the inflammatory, proliferative and remodeling phases of the wound healing program ${ }^{6,10,11}$.

The localization of HFSCs in the mouse skin has been studied using genetic lineage reporter models ${ }^{9,12-14}$ or by mapping endogenous markers such as Sox9 a master regulator of HFSC identity, whose expression has been used extensively to trace HFSCs $^{5,12,15-20}$. With this bona-fide endogenous marker of HFSCs we have studied their spatio-temporal localization in homeostatic and wounded skin as well as in wild-type (WT) and C8cKO mice (Figure 1A).

HFSCs and their progeny have been reported in the epidermis robustly by $2-$ 5 days following wounding ${ }^{9,21}$, but whether changes in HFSC activation can be observed early after injury have surprisingly not been reported. In order to fill this gap in our understanding of the initial cellular reaction of HFSCs to a wound, we chose to examine the behavior of these cells in the 8 - 16 hour time-frame when proliferation in the HFSC niche has yet to begin (Supplementary figure 1A). Typically, under homeostatic conditions in the adult mice at the telogen stage of the hair cycle, HFSCs expressing Sox9 are clustered at the base of the hair follicle in their niche known as the bulge (Figure 1A). However upon a full thickness excisional wound, some HFSCs migrate upwards from the bulge along the outer layer of the hair follicles to infundibulum (upper part of the hair follicle) and infiltrate the epidermis in 24 hours (Figure 1A). This observation was remarkable as it is generally assumed that proliferation of the HFSCs in their niche would precede migration as to maintain a constant population in the bulge. This data indicates that emigration HFSCs from the bulge precedes proliferation and suggests that this may be a contributing factor to signal the remaining cells to multiply in order to repopulate the depleted niche.

To quantitatively track the changes in HFSC localization upon wounding, we developed an image analysis algorithm which measures the distance of individual HFSCs from the epidermal junction along hair follicles, and calculates their frequency of occurrence in bins of equal dimensions along the length of the hair follicle (Supplementary figure 1B). In Figure 1B, we represent the quantitative distribution of HFSCs along hair follicles as a heatmap, where the regions having larger number of HFSCs are represented in lighter colors. Consistent with both published reports ${ }^{16}$ and with the qualitative localization of Sox9 expressing HFSCs, the highest probability of occurrence of HFSCs is detected in the bulge ( $150-250 \mu \mathrm{m}$ from the epidermis) of hair-follicles in both unwounded and wounded skin. We did not observe a statistically 
significant increase in relocalization of the HFSCs near the infundibulum (within $80 \mu \mathrm{m}$ of the epidermis) at 8 hours after wounding (Supplementary Figure 1C). However, by 16 hours post-wounding, we observed a measurable increase in the frequency of HFSCs close to the infundibulum (Supplementary Figure 1C) and by 24 hours, they are detected at the infundibulum (Figure 1B, Supplementary Figure 1C). Thus we have uncovered a novel phenomenon wherein migration of HFSCs precedes their proliferation in the niche, typically observed in the bulge after 72 hours of wound-healing 22. This observation is similar to the behavior of keratinocytes in the wounded epidermis, where the immediate wound-proximal region of the epidermis has only migrating cells followed by a proliferative zone ${ }^{23,24}$.

To test if the results from the analysis of HFSC migration in response to injury is recapitulated in the genetic model of wound-healing (C8cKO) we mapped the localization of HFSCs in neonatal mice when the skin phenotype develops ${ }^{6,10}$, (Figure 1C). At this stage, the hair follicle is still in morphogenesis when there is no defined bulge and Sox9 expression can be observed along the entire length of the hair follicle ${ }^{20}$ with a substantial accumulation within 50-100 $\mu \mathrm{m}$ of the epidermis. However, in the C8cKO skin, there is a noticeable accrual of HFSCs localizing within the infundibulum region of the hair follicle $(<50 \mu \mathrm{m})$. Using the image analysis algorithm, we have observed a statistically significant increase in the HFSC localization within the infundibulum of the postnatal day 4 (p4) C8cKO skin (Figure 1D). Interestingly, at this p4 timepoint, in the genetic model of wounding, we do not detect any Sox9 expressing HFSCs in the epidermis (Figure 1E). Nevertheless, as the wound healing phenotype advances in postnatal day 6-7 mice, there is a progressive increase in the number of HFSCs detected in the interfollicular epidermis (Figures $1 \mathrm{C}$ and $\mathrm{E}$ ). This result recapitulates the phenomenon observed in the edge of an excisional wound wherein there is increasing amounts of HFSCs in the epidermis beginning one day after wounding and thereafter (Figures $1 \mathrm{~A}$ and $\mathrm{E}$ ).

To further confirm the trend of HFSCs redistribution along hair follicles as migration, we scored for the activation of genes that can impact their chemotactic behavior. Analysis of published transcriptome data obtained from HFSCs isolated from unwounded and wounded skin ${ }^{21}$ yielded a set of genes ontologically associated with cell migration ${ }^{\square}$ (Supplementary Figure 1D). From this dataset, select migration associated genes were tested for their expression at different time points post excisional wounding (Supplementary Figure 1E). This analysis demonstrated a significant upregulation of migratory genes by 8 hours following excisional wounding (Figure 1F). The observation that the HFSC migration gene program is activated (at 8 hours) even before a measurable HFSC flux out of the niche is observed (at 16 hours), supports previous observations that HFSCs activate the wound-response program at the gene expression level considerably before they physically start migrating out of their niche ${ }^{25}$. A similar trend in the expression of the migration genes is seen in the epithelial compartment of the C8cKO skin from p4 mice prior to their entrance into the interfollicular epidermis of the skin (Figure 1F). Altogether, these results suggest that the C8CKO mouse recapitulates both the migratory gene signature as well as the motility 
behavior of the HFSCs in response to an excisional wound. We therefore exploited the C8cKO skin as a platform to dissect the molecular mechanisms regulating the homing behavior of HFSC from the hair follicle to the epidermis.

\section{Caspase-1 is a critical regulator of HFSC migration to the epidermis.}

An important feature that is conserved between the excisional wound and the C8cKO is the activation of the Caspase-1 containing inflammasome. The inflammasome is a macromolecular complex in the cytosol that mediates the unconventional secretion of many inflammatory cytokines including IL-1 $\alpha^{6,10}$. In particular, IL-1 $\alpha$ mediates the reciprocal interactions between epidermal keratinocytes and dermal fibroblasts to promote proliferation of basal epidermal stem cells. Moreover, IL-1 $\alpha$ also mediates the crosstalk between epidermal keratinocytes and resident dendritic epidermal T-cells to promote HFSC proliferation ${ }^{11}$. Consequently, mice lacking caspase-1 $\left(\mathrm{C}^{-/-}\right)$exhibit a delay in wound closure by affecting epithelial stem cell proliferation in different niches of the skin $^{6}$. We find that caspase-1 expression is upregulated as early as 8 hours of wound-healing (Supplementary Figure 2A), a time frame corresponding with the upregulation of HFSC migration genes. Given its effects on proliferation, we hypothesized that caspase-1 may also influence re-epithelialization of the wound by regulating the migration of HFSCs to the epidermis. To test this hypothesis, we analyzed the distribution of HFSCS along the hair follicles in C8cKO skin lacking caspase-1 (C8cKO/ $\left.\mathrm{C1}^{-/-}\right)$and compared it to their localization in C8cKO and WT skins (Figure 2A). The HFSCs found to be epidermally directed (present within the infundibulum) in the C8cKO skin at $\mathrm{p} 4$, is substantially reduced in the $\mathrm{C} 8 \mathrm{cKO} / \mathrm{C}^{-/-}$skin (Figure 2A). Quantitative analysis of HFSC localization along hair follicles in these mice indeed revealed that the increased frequency of HFSCs in the infundibulum $(<50 \mu \mathrm{m}$ from the basal layer of the epidermis) in the C8cKO mice relative to its wild type counterpart is significantly reduced in the absence of Caspase-1 $\left(\mathrm{C} 8 \mathrm{cKO} / \mathrm{C}^{-1 /}\right.$ ) (Figure 2B). This suggests that epidermal chemotaxis of HFSCs is dependent on Caspase- 1. This suggests that the initial chemotaxis of HFSCs towards the epidermis is largely dependent on Caspase-1. We further validated whether these observations from the genetic wound-healing model, are applicable to excisional wounds in the $\mathrm{C} 1^{-/}$mice. In mice lacking caspase-1 wound wound-healing is slower and epidermal thickness is inhibited ${ }^{6}$. Similar to wild-type mice, the largest proportion of HFSCs in the $\mathrm{C}^{-/-}$mice are present in the bulge region (150-250 $\mu \mathrm{m}$ from the epidermis) under homeostatic (unwounded) conditions (Figures 2C, D). Within 16-24 hours of the wound-healing program, unlike the wild-type skin, we do not observe migration of HFSCs into the infundibulum $(<50 \mu \mathrm{m})$ in the $\mathrm{C}^{-/}$mice (Figure 2D, Supplementary Figure 2B). The defect in migration is even more pronounced in the $\mathrm{C}^{-/-}$background at later timepoints as the HFSCs begin to populate the epidermis. At 24 hours of wound-healing, in the wild type skin, the number of HFSCs in the epidermis proximal to wound edge is 2.5 times higher than distal hair follicles $500 \mu \mathrm{m}$ from the wound bed (Figure 2E). This is consistent with many reports that cellular activity is spatially restricted to an area immediately adjacent to the site of tissue damage ${ }^{9,23,26}$. However, in the wounded skin 
lacking caspase-1, the number of HFSCs in the epidermis at the proximal wound edge is comparable to the numbers found distal to the wound site (Figure 2E). This data clearly demonstrates that the robust migration of HFSCs into the epidermis during wound-healing is critically dependent upon the presence of Caspase-1. We next examined whether the effect of caspase-1 on HFSC migration is a manifestation of its impact on the same migratory genes that are upregulated in both the genetic and excisional wound healing models (Figure 1F). Expression of several of the migration associated genes is reduced in the $\mathrm{C} 8 \mathrm{CKO} / \mathrm{C}^{-/-}$epidermis and the $\mathrm{C} 1^{-/-}$wound when compared to the $\mathrm{C} 8 \mathrm{cKO}$ epidermis and WT wound respectively (Figure 2F). Interestingly, the upregulation of these migration associated genes recover at later timepoints, thereby demonstrating a later, caspase-1 independent phase of HFSC migration (16 - 24 hours, Supplementary Figure 2C). Consistent with this, though there is a significant delay in early stages of HFSC emigration to the epidermis we ultimately observe a large number of epidermal HFSCs by 2-3 days of wound-healing in the $\mathrm{C}^{-1-}$ mice (Supplementary Figure 2D). This suggests that caspase- 1 is a critical initial catalyst of HFSC homing into the epidermis that is further supplemented by alternative signaling pathways to reinforce stem cell chemotaxis into this tissue.

\section{HFSC migration to the epidermis is mediated by soluble chemotactic cues.}

An important question that arises from this observation is the mechanism by which caspase- 1 regulates HFSC migration. The canonical function of caspase-1 as an inflammatory molecule is well studied in immune cells as well as in non-myeloid cells like keratinocytes ${ }^{27,28}$. Caspase-1 is expressed as a zymogen which undergoes activation upon binding to intercellular complexes known as inflammosomes. The activation of Caspase- 1 is required for the cleavage and release of cytokines and several molecules that mediate diverse cellular processes such as survival, inflammation and tissue repair ${ }^{29}$. We wondered whether it is this caspase- 1 dependent secretome from stressed keratinocytes that is regulating the HFSC chemotaxis observed in both the excisional and genetic model of wound-healing. To test if soluble chemotactic factors released from wounded-keratinocytes regulate HFSC migration, we utilized a transwell migration assay with wild-type isolated HFSCs ${ }^{30}$ and scored their chemotactic index in response to conditioned media $(\mathrm{CM})$ prepared from either wild-type or C8cKO epidermis. In comparison to wild-type epidermal CM, C8cKO CM could significantly enhance HFSC migration (Figure $3 \mathrm{~A}$ ). However, epidermal CM from $\mathrm{C} 8 \mathrm{cKO} / \mathrm{C}^{-1-}$ mice did not exhibit any HFSC migration promoting activity. This result supports the hypothesis that soluble cues released from wounded keratinocytes stimulates HFSC migration in a Caspase-1 dependent manner. Importantly, the chemotactic cues found in the C8cKO CM does not appear to be a generic stimulus for epithelial stem cell migration as the CM does not enhance chemotaxis of primary mouse epidermal keratinocytes (Supplementary Figure 3A). This suggests that the factor(s) controlling epidermal homing of HFSCs are unique from the classical regulators of epidermal keratinocyte motility ${ }^{31}$. This unconventional mechanism partly explains the elusive nature of understanding wound-induced HFSC chemotaxis.

Keratinocyte stress leads to caspase- 1 activation and release of IL- $1 \alpha$, which 
we previously reported to be important to stimulate both epidermal stem cell and HFSC proliferation ${ }^{11}$. IL-1 $\alpha$ mediates the reciprocal interactions of epidermal keratinocytes and dermal fibroblasts to induce proliferation of keratinocytes of the basal layer of the epidermis ${ }^{10}$. We have also found that $\mathrm{IL}-1 \alpha$ mediates the crosstalk between epidermal keratinocytes and resident dendritic epidermal T-cells that ultimately results in the stimulation of HFSC proliferation ${ }^{11}$. Despite the importance of IL- $1 \alpha$ signaling on the proliferation of different epithelial stem cell pools in the skin, we previously observed that it does not affect the migration of the HFSCs into the epidermis ${ }^{11}$. In light of the fact that the previous tests of the usual candidates of chemotactic factors did not impact directed cell migration of HFSCs, we focused on the often overlooked fact is that Caspase- 1 activation also results in its own unconventional secretion ${ }^{10,29,32,33}$. This can be in response to stress stimuli such as UV irradiation or wounding of keratinocytes $^{10,29,34}$. Though the secretion of caspase-1 is a well-documented phenomenon, the function of this extracellular caspase-1 is largely unknown. We found that epidermal keratinocytes are a major source of extracellular caspase-1 (Figure 3B). When we mimicked the wound-reaction in human keratinocytes by simply knocking down caspase-8 the cells responded by increasing the release of caspase-1, which we measured in the conditioned media (Figure 3B). We probed whether the presence of extracellular caspase-1 indicates an unknown activity of the molecule in mediating the extensive network of intercellular crosstalk that would induce HFSC chemotaxis in a wound microenvironment. To test this hypothesis, C8cKO-CM was immunodepleted of caspase-1 and its effect on HFSC migration was investigated (Figure 3C). Resonating with the data in Figure 3A, we find that the removal of extracellular caspase- 1 from the $\mathrm{CM}$ results in the loss of migration in HFSCs (Figure $3 \mathrm{C}$ ). The intracellular role of caspase-1 as part of the inflammasome to mediate unconventional secretion of proteins is primarily dependent on its catalytic function ${ }^{29}$. Hence we tested whether the catalytic activity of extracellular caspase-1 present in C8cKO-CM is required for HFSC chemotaxis. Upon inhibiting caspase-1 activity in the conditioned media using a cell impermeable inhibitor, we found no reduction in migration (Figure 3D). Altogether, these results suggest that extracellular caspase- 1 has a novel, non-catalytic role of promoting the chemotactic migration of HFSCs.

\section{Recombinant Caspase-1 is sufficient to induce HFSC migration to the epidermis:}

Our data demonstrates that extracellular caspase-1 is necessary to promote the directed migration of HFSCs, but is it sufficient to trigger this process? To test this, we bacterially expressed and purified (Supplementary Figure 4A) murine pro-Caspase-1 having catalytic function (rCasp-1 WT) or mutated at cysteine 284 (C284A) ${ }^{35}$ to abolish its catalytic activity (rCasp-1 C284A). The identity of the recombinant proteins was confirmed by mass spectrometry and their catalytic activity was verified by a substrate cleavage assay (Supplementary Figure 4B). Whereas the purified rCasp-1 WT was efficient in cleaving its substrate, the rCasp-1 C284A is indeed catalytically dead. We then tested whether the wild-type or the mutant protein is sufficient to stimulate HFSC chemotaxis. In comparison to control treated samples, both the rCasp-1 WT and rCasp- 
1C284A proteins had equal potential to induce chemotaxis of HFSCs (Figure 4A). This supports our earlier observation based on pharmacological data that the catalytic function is dispensable for Caspase- 1 to operate as a HFSC chemoattractant (Figure 3D). Moreover, the capacity of Caspase-1 to induce migration in HFSCs is independent of their proliferation, as measured by the response of mitomycin $\mathrm{C}$ treated HFSCs to rCasp-1C284A in comparison to untreated HFSCs (Figure 4B). This supports the observations made in Figures 1 and 2, where we found that the early migratory wave (at 16 - 24 hours) of HFSCs in a caspase-1 dependent manner is independent of proliferation in the niche. This finding is consistent with recent reports that during the reepithelialization process of wound healing, migrating cells and proliferating cells are two spatially distinct populations ${ }^{23,24}$

Extending on this in vitro observations, we assayed for the capacity of rCasp$1 \mathrm{C} 284 \mathrm{~A}$ to induce HFSC migration to the epidermis in skin explants from $\mathrm{C}^{-1-}$ mice. Indeed, we find that there is relocalization of HFSCs to the interfollicular epidermis upon treatment with rCasp-1C284A (Figure 4C). Given this unusual catalytic-independent function of caspase-1 as a chemoattractant, we next investigated which domain is responsible for this novel activity. Caspase- 1 is comprised of an $\mathrm{N}$-terminal Caspase Activation Recruitment Domain (CARD), followed by the $\mathrm{p}-20$ and $\mathrm{p}-10$ domains ${ }^{35}$. Autoproteolysis results in the separation of these individual domains, followed by the heterodimerization of the p20 and p10 domains into the catalytically active form. Since the catalytic activity is not required for inducing HFSC migration, we focused on the CARD domain which is the protein-protein interaction region of caspase- ${ }^{36}$. The murine Caspase-1 CARD was tagged with polyhistidine, bacterially expressed and purified by affinity chromatography (Supplementary Figure 4C), and then tested for its ability to induce HFSC migration. We found that the CARD of caspase-1 was sufficient to induce HFSC migration in vitro (Figure 4D). To test whether this phenomenon is likewise conserved in the 3D organoid system, we treated explants of full thickness skin punch biopsies with purified CARD domain. This treatment revealed that CARD is sufficient to induce the migration of HFSCs upwards into the infundibulum of the hair follicle (Figure 4E). These results indicate that extracellular Caspase-1 mediates its non-canonical chemotactic function largely by its CARD.

\section{Caspase-1 is a regulator of stem cell responses in the skin during UV damage:}

Our data demonstrates that wounding leads to the release of extracellular caspase-1 from stressed keratinocytes, which acts as a chemotactic factor to elicit HFSC migration to the epidermis. However, this is not the only scenario reported to exhibit the secretion of caspase-1. Skin carcinomas ${ }^{37,38}$ and other inflammatory skin diseases such as psoriasis ${ }^{8}$, atopic dermatitis ${ }^{39,40}$ and sunburn ${ }^{7}$, are all marked by caspase-1 activation. Thus we examined whether extracellular caspase-1 mediated HFSC migration into the epidermis is a conserved phenomenon that may explain the epithelial hyperplasia associated with these pathologies. In particular, we investigated whether UV irradiation could induce HFSC migration in vitro and ex vivo. Since UV irradiated mouse keratinocytes release caspase-1 ${ }^{29,34}$, we tested whether conditioned media collected within 4 hours of irradiation is capable of stimulating HFSC chemotaxis. 
We found that CM of UV irradiated keratinocytes can significantly enhance HFSC chemotaxis compared to $\mathrm{CM}$ from non-irradiated keratinocytes (Figure 5A). We also examined whether UV irradiation of mouse skin explants displayed epidermal homing behavior of HFSCs in a caspase-1 dependent manner. Within 16 hours of UV irradiation of mouse skin explants, we observed HFSCs migrating upwards along hair-follicles to the epidermis in wild-type skin (Figure 5B). However, there was a substantial reduction in the number of HFSCs homing into the epidermis in $\mathrm{C}^{-1-}$ skin explants exposed to UV irradiation (Figures 5B and C).

Altogether a model emerges wherein Caspase-1 is an important mediator of both inflammatory signaling as well as stem cell migration in wound-healing and other skin pathologies characterized by inflammation. This novel chemotactic function is mediated by the extracellular caspase-1, more specifically by its protein-protein interaction domain CARD. Our work elucidates the role of extracellular caspase-1 as a bulge specific HFSC chemoattractant but it might have similar roles on other stem cells in skin appendages known to participate in wound-healing.

\section{Main figure legends.}

\section{Figure 1: Epidermal knock-out of Caspase-8 (C8cKO) recapitulates HFSC migration to the epidermis:}

A. Localization of HFSCs marked by Sox9 (red) in adult skin where the hair follicle and epidermis are marked by keratin-5 (green) in unwounded (UW), line marking the bulge; and at 8,16 , and 24 hours of wound healing $(\mathrm{W})$. Boxed region marks the infundibulum (inset).

B. Probability distribution of HFSCs along the hair follicle in unwounded (UW) and wounded (W) skin at 8, 16 and 24 hours post wounding, represented as a heat-map with distance from the epidermis represented on the y-axis. Statistics of frequency change between UW and $W$ conditions has been calculated from $n=3$ mice of each genotype at each condition (Supplementary Figure 1C).

C. HFSC localization in the skin of wild-type (WT) and caspase-8 conditional knock-out (C8cKO) mice of age postnatal days 4 (p4) and 6 (p6). Inset showing infundibulum at $\mathrm{p} 4$ and interfollicular epidermis at $\mathrm{p} 6$.

D. Probability distribution of HFSCs along hair follicles in the skin of WT and C8cKO mice at p4. Statistics of frequency change between WT and C8cKO has been calculated from $n=3$ mice of each genotype. Green box marks the region of hair follicles where the localization of HFSCs have significantly changed.

E. HFSC numbers analysed from the interfollicular epidermis (50 by $50 \mu \mathrm{m}^{2}$ regions) in C8cKO mice at postnatal days 4, 6 and 7 (blue bars); and compared to the wound edge epidermis at 16, 24 and 72 hours of wound-healing (W) (green bars). Mean and S.E.M is calculated from $n=3$ animals at each condition.

F. Expression analysis of HFSC specific wound activated migration genes from unwounded (UW, green) and wounded skin (W, blue) at 8 hours of wound-healing) 
and compared to that measured in WT (green hatch) and C8cKO (blue hatch) p4 epidermis and hair follicles. UW and $W: n=6$, p4 WT and C8cKO: $n=5$. Error bars represent S.E.M.

Figure 2: Caspase-1 regulates HFSC migration to the epidermis:

A. Panel showing the localization of HFSCs (Sox9, red) in WT, C8cKO and C8cKO/C1 $1^{-1-}$ skin at postnatal day 4. Epidermis and hair follicles are marked by $\mathrm{K} 5$ expression (green). Inset showing the infundibulum of the hair follicle.

B. Probability distribution of HFSCs along hair follicles analyzed from WT, C8cKO and $\mathrm{C} 8 \mathrm{cKO} / \mathrm{C}^{-1-}$ skin at $\mathrm{p} 4$. Statistics of frequency change between genotypes has been calculated from $n=3$ mice of each genotype. Green box marks the region of hair follicles where the localization of HFSCs have significantly changed between C8cKO and $\mathrm{C} 8 \mathrm{cKO} / \mathrm{C} 1^{-/-}$skin.

C. HFSC localization (Sox9, red) in the wound proximal region of WT and $\mathrm{C}^{-/-}$mice at 16 and 24 hours of wound-healing.

D. Probability distribution of HFSCs along hair follicles at 16 and 24 hours of woundhealing in WT and $\mathrm{C1}^{-/-}$wounds (W) and respective unwounded (UW) skin. Statistics of frequency change between UW and $W$ conditions has been calculated from $n=3$ mice of each genotype at each condition (Supplementary Figure 2B).

E. Quantification of migrated HFSCs into the epithelial lip at 24 hours post wounding in WT (blue, $n=5)$ and Caspase- $1^{-/-}(\mathrm{red}, \mathrm{n}=4)$ wounds, as estimated from 100 by 100 $\mu \mathrm{m}^{2}$ boxes.

F. Expression analysis of HFSC specific migration genes at 8 hours of wound-healing in WT (blue, $\mathrm{n}=6)$ and Caspase $-1^{-/}(\mathrm{red}, \mathrm{n}=4)$ mice in comparison to the same in the epidermis and hair follicles of $\mathrm{C} 8 \mathrm{cKO}$ (blue hatch) and $\mathrm{C} 8 \mathrm{cKO} / \mathrm{C} 1^{-/-}$skin (red hatch). Error bars represent S.E.M.

Figure 3 : HFSC migration to the epidermis is mediated by soluble chemotactic cues.

A. HFSC migration in a transwell Boyden chamber assay to conditioned media from C8cKO epidermis (C8cKO), C8cKO-Caspase-1 null epidermis $\left(\mathrm{C} 8 \mathrm{cKO} / \mathrm{C1}^{-1-}\right)$ in comparison to that from wild-type epidermis (WT). Data represented from $n=4$.

B. Detection of caspase-1 in the lysate (Lys) and conditioned media (CM) from Hacat cells transduced with scrambled shRNA, or shRNA against Caspase-8 in knockdown cells (KD). Caspase- 8 knock-down is $>80 \%$.

C. HFSC migration in response to $\mathrm{C} 8 \mathrm{cKO}$ epidermal conditioned media with (C8cKO + Casp-1 Ab) or without (C8cKO) extracellular Caspase-1 removed by immunoprecipitation, and the corresponding $\operatorname{lgG}$ control (C8cKO + $\operatorname{lgG})$ in comparison to WT conditioned media. Data is represented from $n=4$ samples.

D. HFSC migration in response to inhibition of catalytic activity of extracellular caspase1 present in the C8cKO epidermal conditioned media (C8cKO + C1inh) compared to vehicle treated C8cKO and WT epidermal conditioned media. Data represented from $\mathrm{n}=3$ samples. Error bars represent S.E.M.

\section{Figure 4: Recombinant Caspase-1 is sufficient to induce HFSC migration to the}




\section{epidermis.}

A. HFSC chemotaxis to recombinant Caspase-1 proteins; wild-type (rCasp-1 WT), catalytic dead (rCasp-1 C284A) in comparison to vector proteins control (vector). $n=$ 4.

B. Chemotaxis of proliferating and non-proliferating (upon Mitomycin treatment, $+\mathrm{MC}$ ) HFSCs to rCasp-1 C284A in comparison to vector control. $n=3$.

C. HFSC localization (Sox9, red) in Caspase- $1^{-/}$skin explant treated with rCasp-1 $\mathrm{C} 284 \mathrm{~A}$ and vector control. Representative images and quantification of number of HFSCs in the epidermis from 100 by $100 \mu m^{2}$ boxes. $n=3$ mice.

D. Analysis of HFSC chemotaxis to recombinant Caspase-1 CARD in comparison to buffer control. $n=3$. Date shows mean and S.E.M. for all experiments.

E. Representative image showing the migration of HFSCs to the infundibulum upon application of CARD.

Figure 5: Caspase-1 regulates UV irradiation induced HFSC migration to the epidermis:

A. HFSC migration in response to conditioned media from control or UV treated (UV$\mathrm{CM})$ primary mouse keratinocytes. $n=3$.

B. HFSC migration towards the epidermis visualized in WT and $\mathrm{C}^{-1-}$ skin explants in untreated or UV treated conditions with Sox9 staining (red). Inset showing interfollicular epidermis.

C. Quantification of HFSCs in the epidermis in the control and UV treated skin explants from WT and $C 1^{-/-}$mice. $n=3$ of each genotype. Date shows mean and S.E.M. for all experiments.

\section{Author Contributions:}

S.G. conceptualized and performed experiments, evaluated and analysed data and wrote the manuscript. N.P. prepared RNA from experimental samples, and performed the gene expression analysis, S.K. developed the image analysis algorithm for measuring HFSC migration, A.S.A cloned, expressed and purified all the recombinant proteins, R.D. analysed RNA-seq data, A.D. performed the UV irradiation of explant experiment, A.B. created the Caspase-8 knock-down cell line, S.P. performed cryosectioning and immunostaining, C.J. contributed to hypothesis development and manuscript preparation.

Acknowledgements: This work is supported by the Wellcome Trust - DBT India Alliance Early Career Fellowship awarded to Subhasri Ghosh. Animal work is supported by the National Mouse Research Resource (NaMoR) grant (102/IFD/SAN/5003/20172018) from DBT.

\section{Reference:}


1. Laird, D. J., von Andrian, U. H. \& Wagers, A. J. Stem cell trafficking in tissue development, growth, and disease. Cell 132, 612-30 (2008).

2. Goodell, M. a., Nguyen, H. \& Shroyer, N. Somatic stem cell heterogeneity: diversity in the blood, skin and intestinal stem cell compartments. Nat. Rev. Mol. Cell Biol. 16, 299-309 (2015).

3. Ge, Y. \& Fuchs, E. Stretching the limits: From homeostasis to stem cell plasticity in wound healing and cancer. Nat. Rev. Genet. 19, 311-325 (2018).

4. Dekoninck, S. \& Blanpain, C. Stem cell dynamics, migration and plasticity during wound healing. Nat. Cell Biol. 21, 40-43 (2019).

5. Plikus, M. V et al. Epithelial stem cells and implications for wound repair. Semin. Cell Dev. Biol. 23, 946-53 (2012).

6. Lee, D.-J. et al. Regulation and Function of the Caspase-1 in an Inflammatory Microenvironment. J. Invest. Dermatol. 135, 1-31 (2015).

7. Faustin, B. \& Reed, J. C. Sunburned skin activates inflammasomes. Trends Cell Biol. 18, 4-8 (2007).

8. Johansen, C., Moeller, K., Kragballe, K. \& Iversen, L. The Activity of Caspase-1 Is Increased in Lesional Psoriatic Epidermis. J. Invest. Dermatol. 127, 2857-2864 (2007).

9. Ito, M. et al. Stem cells in the hair follicle bulge contribute to wound repair but not to homeostasis of the epidermis. Nat. Med. 11, 1351-1354 (2005).

10. Lee, P. et al. Dynamic expression of epidermal caspase 8 simulates a wound healing response. Nature 458, 519-23 (2009).

11. Lee, $\mathrm{P}$. et al. Stimulation of hair follicle stem cell proliferation through an IL-1 dependent activation of gd T-cells. Elife 6, 1-20 (2017).

12. Adam, R. C. et al. Pioneer factors govern super-enhancer dynamics in stem cell plasticity and lineage choice. Nature 521, 366-70 (2015).

13. Morris, R. J. et al. Capturing and profiling adult hair follicle stem cells. Nat. Biotechnol. 22, 411-7 (2004).

14. Kretzschmar, K. \& Watt, F. M. Lineage tracing. Cell 148, 33-45 (2012).

15. Larsimont, J. et al. Sox9 Controls Self-Renewal of Oncogene Targeted Cells and Links Tumor Initiation and Invasion Article Sox9 Controls Self-Renewal of Oncogene Targeted Cells and Links Tumor Initiation and Invasion. Cell Stem Cell 17, 60-73 (2015).

16. Hsu, Y.-C., Pasolli, H. A. \& Fuchs, E. Dynamics between stem cells, niche, and progeny in the hair follicle. Cell 144, 92-105 (2011).

17. Wong, S. Y. \& Reiter, J. F. Wounding mobilizes hair follicle stem cells to form tumors. Proc. Natl. Acad. Sci. U. S. A. 108, 4093-4098 (2011).

18. Fuchs, Y. et al. Sept4/ARTS Regulates Stem Cell Apoptosis and Skin Regeneration. Science 341, 286-290 (2013).

19. Nowak, J. a., Polak, L., Pasolli, H. A. \& Fuchs, E. Hair follicle stem cells are specified and function in early skin morphogenesis. Cell Stem Cell 3, 33-43 (2008).

20. Vidal, V. P. I. et al. Sox9 Is Essential for Outer Root Sheath Differentiation and the Formation of the Hair Stem Cell Compartment. Curr. Biol. 15, 1340-1351 (2005).

21. Ge, Y. et al. Stem Cell Lineage Infidelity Drives Wound Repair and Cancer. Cell 169, 636-642.e14 (2017).

22. Mardaryev, A. N. et al. Lhx2 differentially regulates Sox9, Tcf4 and Lgr5 in hair follicle stem cells to promote epidermal regeneration after injury. Development 138, 48434852 (2011).

23. Aragona, M. et al. Defining stem cell dynamics and migration during wound healing in 
mouse skin epidermis. Nat. Commun. 8, (2017).

24. Park, S. et al. Tissue-scale coordination of cellular behaviour promotes epidermal wound repair in live mice. Nat. Cell Biol. 19, (2017).

25. Joost, S. et al. Single-Cell Transcriptomics of Traced Epidermal and Hair Follicle Stem Cells Reveals Rapid Adaptations during Wound Healing Article Single-Cell Transcriptomics of Traced Epidermal and Hair Follicle Stem Cells Reveals Rapid Adaptations during Wound Healing. CellReports 25, 585-597.e7 (2018).

26. Mascré, G. et al. Distinct contribution of stem and progenitor cells to epidermal maintenance. Nature 489, 257-62 (2012).

27. Sun, Q. \& Scott, M. J. Caspase-1 as a multifunctional inflammatory mediator $\square$ : noncytokine maturation roles. J. Leukoc. Biol. 100, 961-967 (2016).

28. Yazdi, A. S., Drexler, S. K. \& Tschopp, J. The Role of the Inflammasome in Nonmyeloid Cells. J. Clin. Immunol. 4, 623-627 (2010).

29. Keller, M., Rüegg, A., Werner, S. \& Beer, H. D. Active Caspase-1 Is a Regulator of Unconventional Protein Secretion. Cell 132, 818-831 (2008).

30. Wu, X. et al. Skin stem cells orchestrate directional migration by regulating microtubule-ACF7 connections through GSK3 $\beta$. Cell 144, 341-352 (2011).

31. Seeger, M. a. \& Paller, A. S. The Roles of Growth Factors in Keratinocyte Migration. Adv. Wound Care 4, 213-224 (2015).

32. Shamaa, O. R., Mitra, S., Gavrilin, M. A. \& Wewers, M. D. Monocyte caspase-1 is released in a stable, active high molecular weight complex distinct from the unstable cell lysate-activated caspase-1. PLoS One 10, 1-21 (2015).

33. Baroja-Mazo, A. et al. The NLRP3 inflammasome is released as a particulate danger signal that amplifies the inflammatory response TL - 15. Nat. Immunol. 15, 738-748 (2014).

34. Feldmeyer, L. et al. The Inflammasome Mediates UVB-Induced Activation and Secretion of Interleukin-1 $\beta$ by Keratinocytes. Curr. Biol. 17, 1140-1145 (2007).

35. Broz, P., Von Moltke, J., Jones, J. W., Vance, R. E. \& Monack, D. M. Differential requirement for caspase-1 autoproteolysis in pathogen-induced cell death and cytokine processing. Cell Host Microbe 8, 471-483 (2010).

36. Park, H. H. Caspase recruitment domains for protein interactions in cellular signaling ( Review ). Int. J. Mol. Med. 43, 1119-1127 (2019).

37. Karki, R. \& Kanneganti, T. Diverging inflammasome signals in tumorigenesis and potential targeting. Nat. Rev. Cancer 19, 197-214 (2019).

38. Drexler, S. K. et al. Tissue-specific opposing functions of the inflammasome adaptor ASC in the regulation of epithelial skin carcinogenesis. Proc. Natl. Acad. Sci. 109, 18384-18389 (2012).

39. Li, C. et al. Development of atopic dermatitis-like skin disease from the chronic loss of epidermal caspase-8. Proc. Natl. Acad. Sci. U. S. A. 107, 22249-54 (2010).

40. Antonopoulos, C. et al. Functional caspase-1 is required for Langerhans cell migration and optimal contact sensitization in mice. J. Immunol. 166, 3672-7 (2001).

41. Li, P. et al. Mice deficient in IL-1b-converting enzyme are defective in production of mature IL-1b and resistant to endotoxic shock. Cell 80, 401-411 (1995).

42. Nowak, J. A. \& Fuchs, E. Isolation and Culture of Epithelial Stem Cells. Methods Mol. Biol. 482, 215-232 (2009).

43. Bolger, A. M., Lohse, M. \& Usadel, B. Trimmomatic: A flexible trimmer for Illumina sequence data. Bioinformatics 30, 2114-2120 (2014).

44. Trapnell, C. et al. Differential gene and transcript expression analysis of RNA-seq experiments with TopHat and Cufflinks. Nat. Protoc. 7, 562-578 (2013). 


\section{Figure 1}

bioRxiv preprint doi: https://doi.org/10.1101/548529; this version posted January 24, 2020. The copyright holder for this preprint (which was not

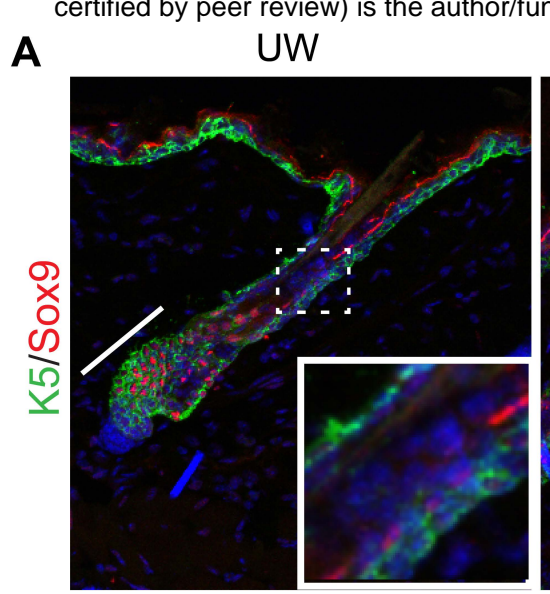

8 hour W

16 hour W

24 hour W
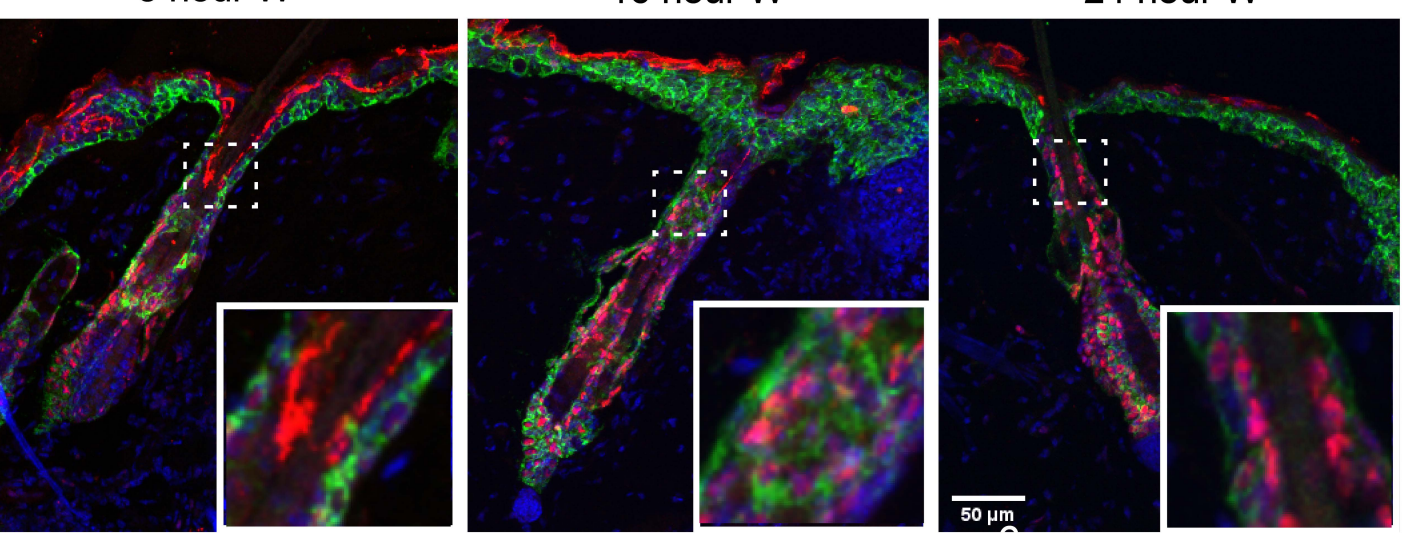

B

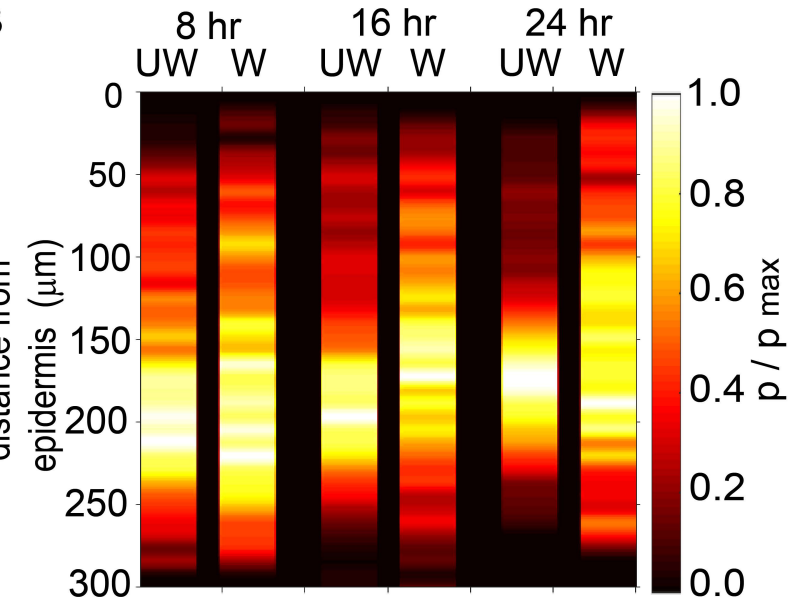

C WT

C8cKO

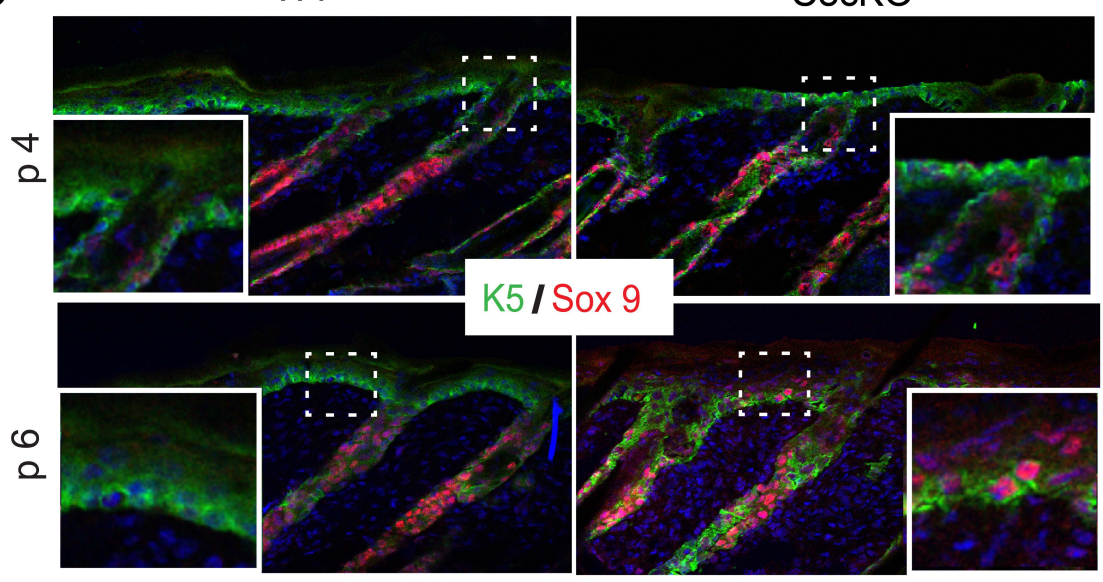

D

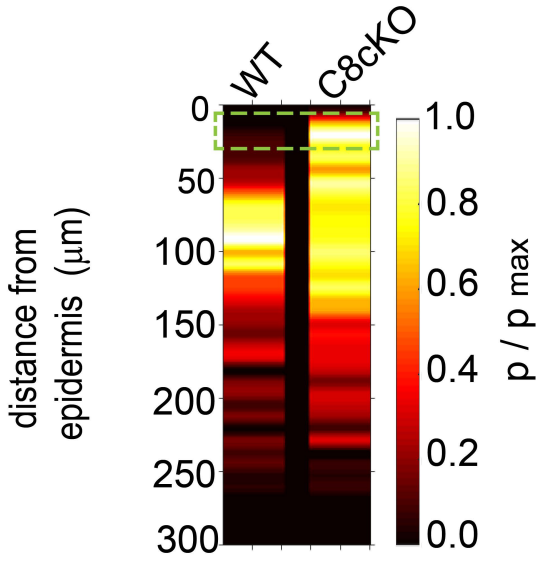

E
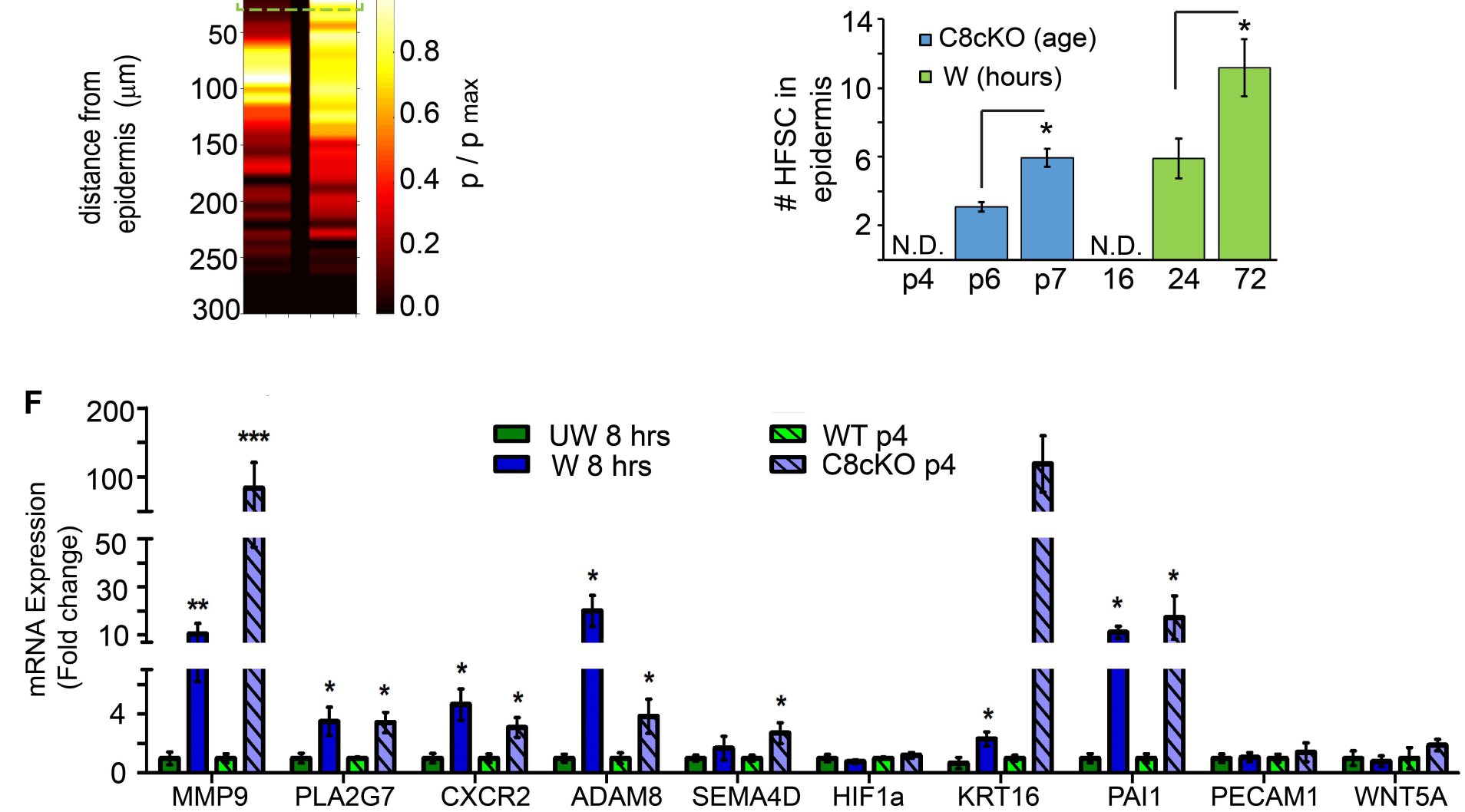
Figure 2

A
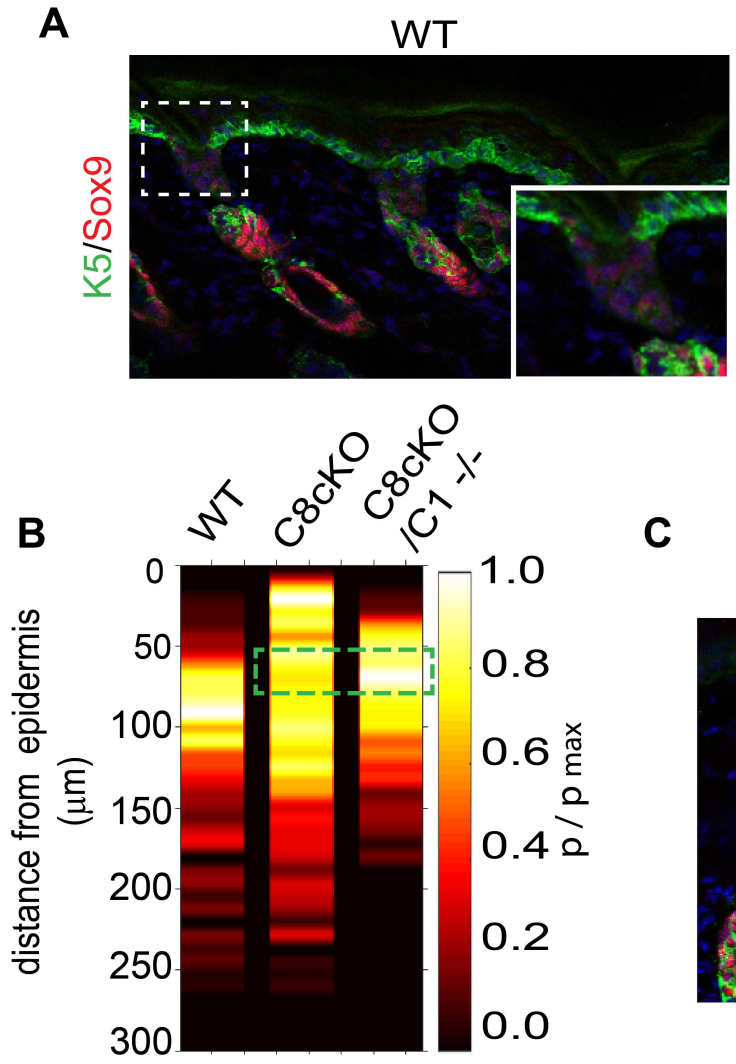

D
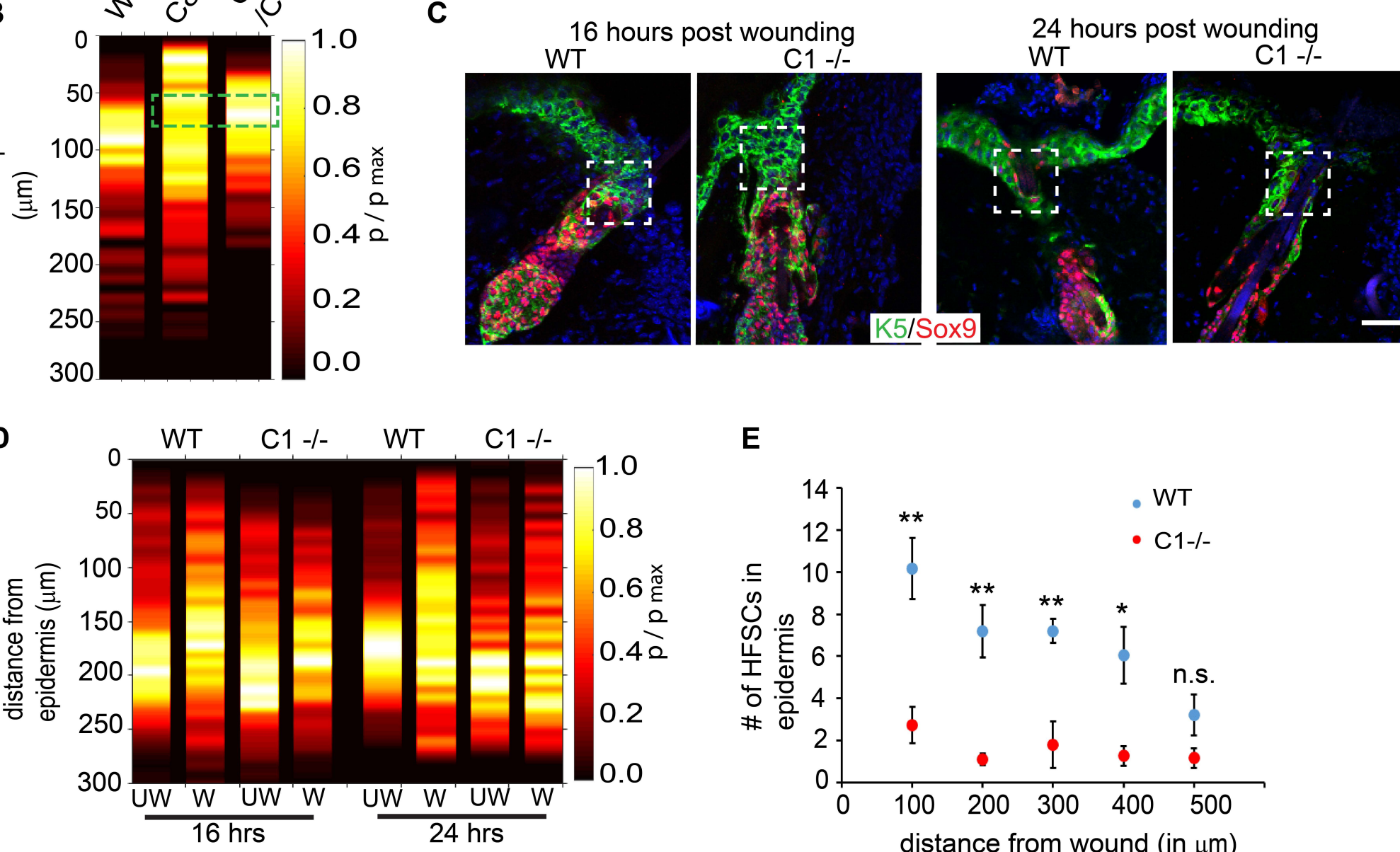

E

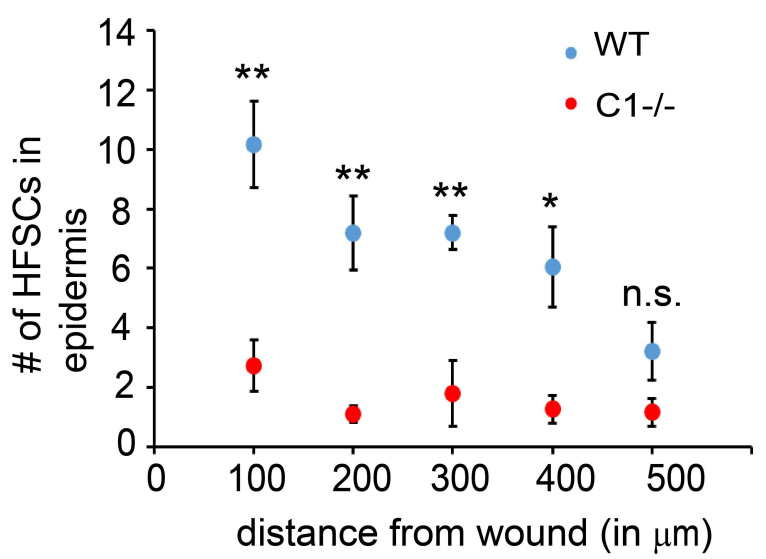

F

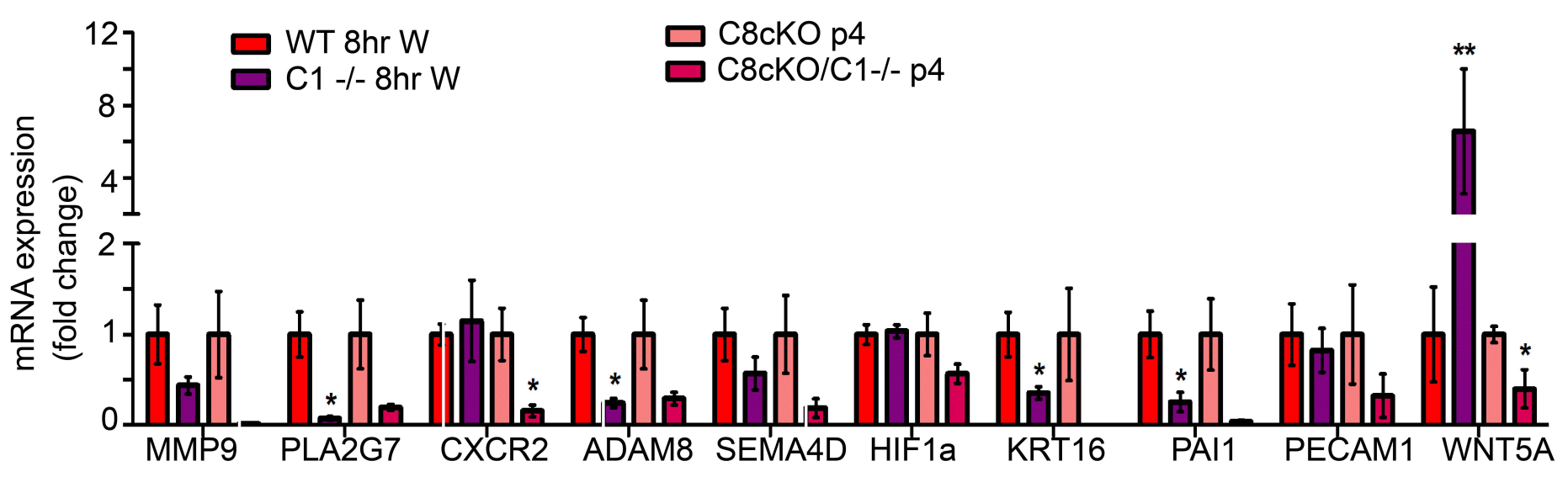




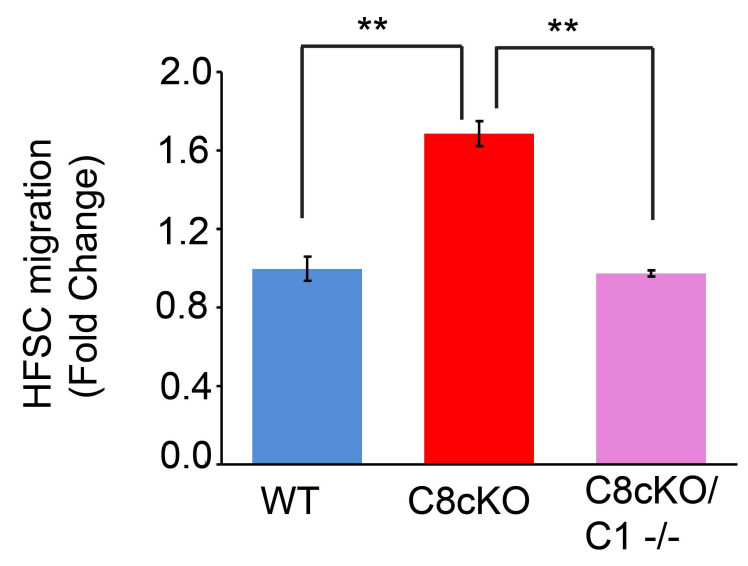

B

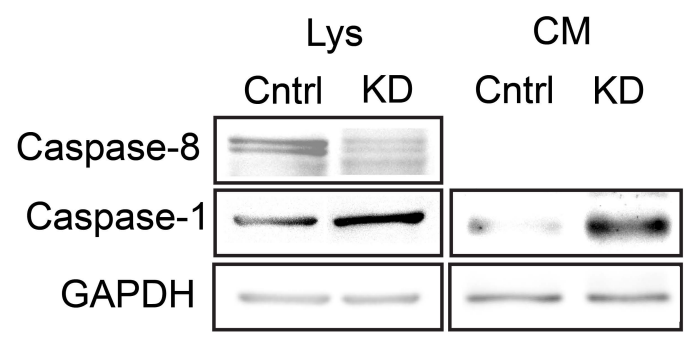

C

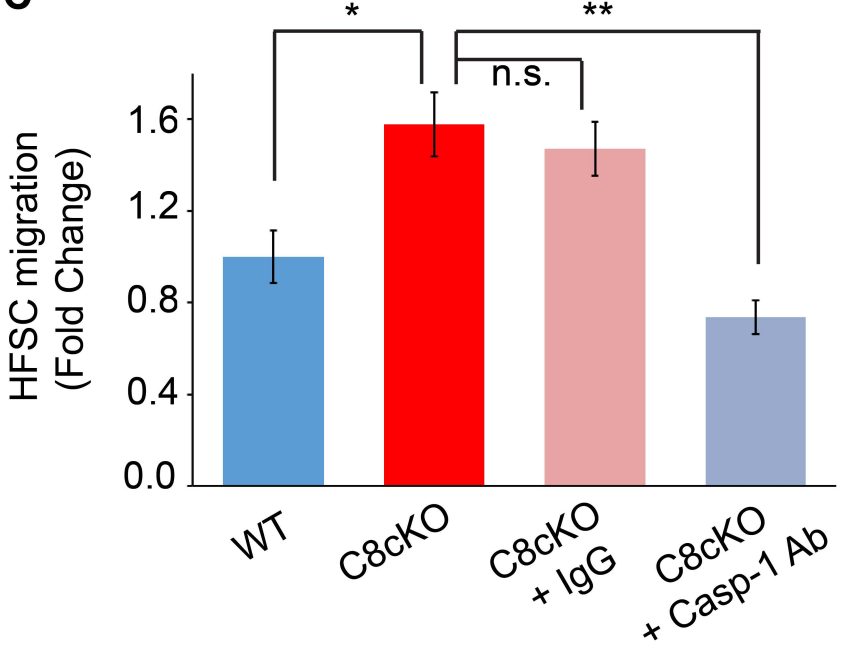

D

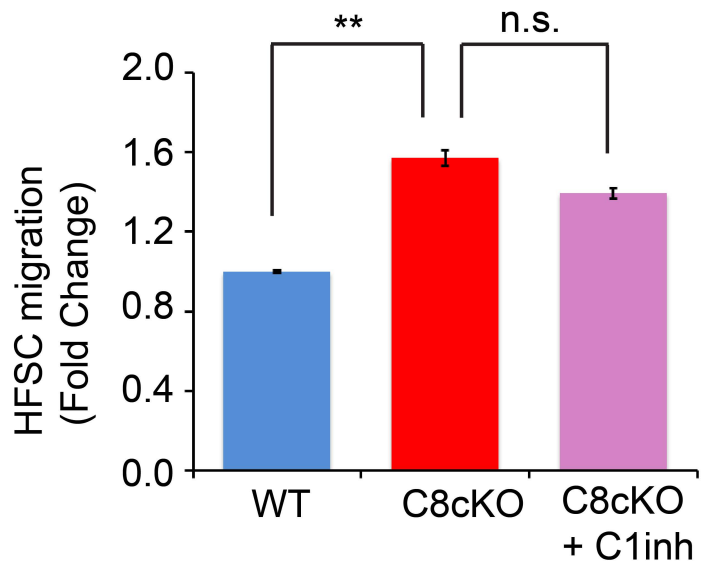


Figure 4

A

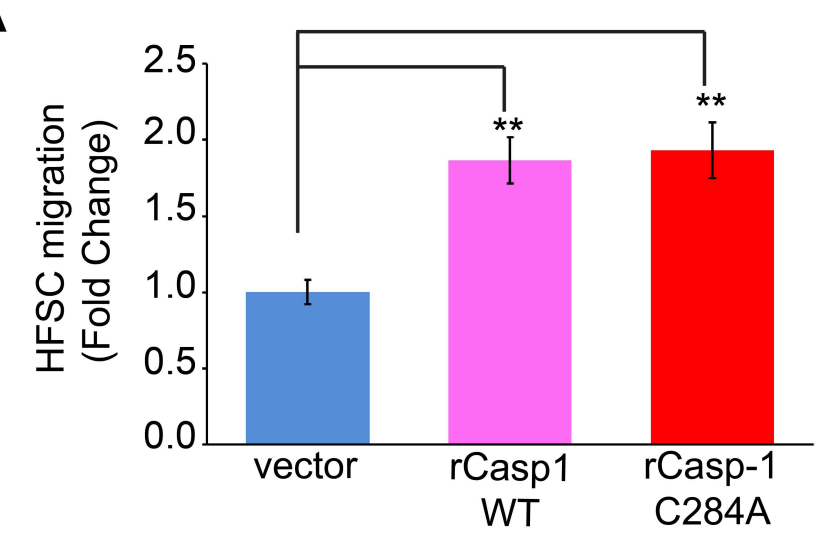

B

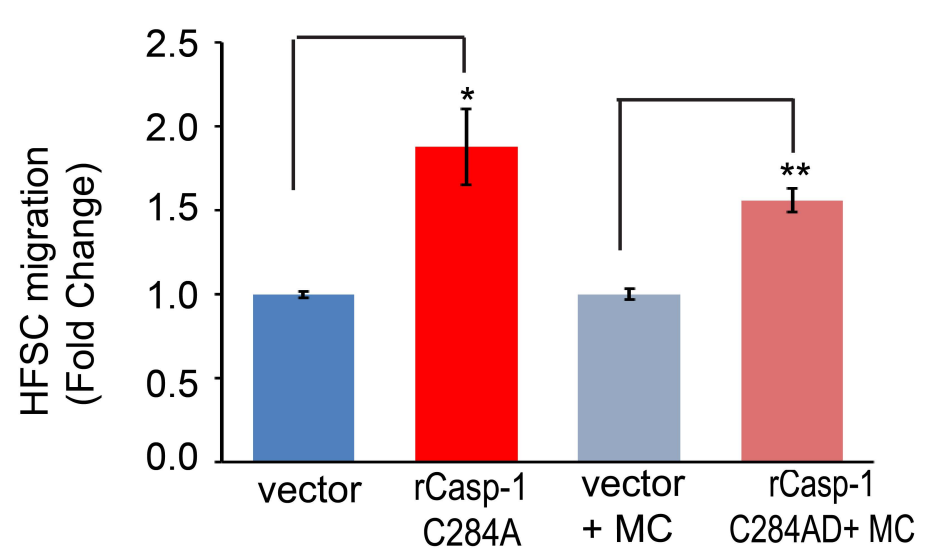

C
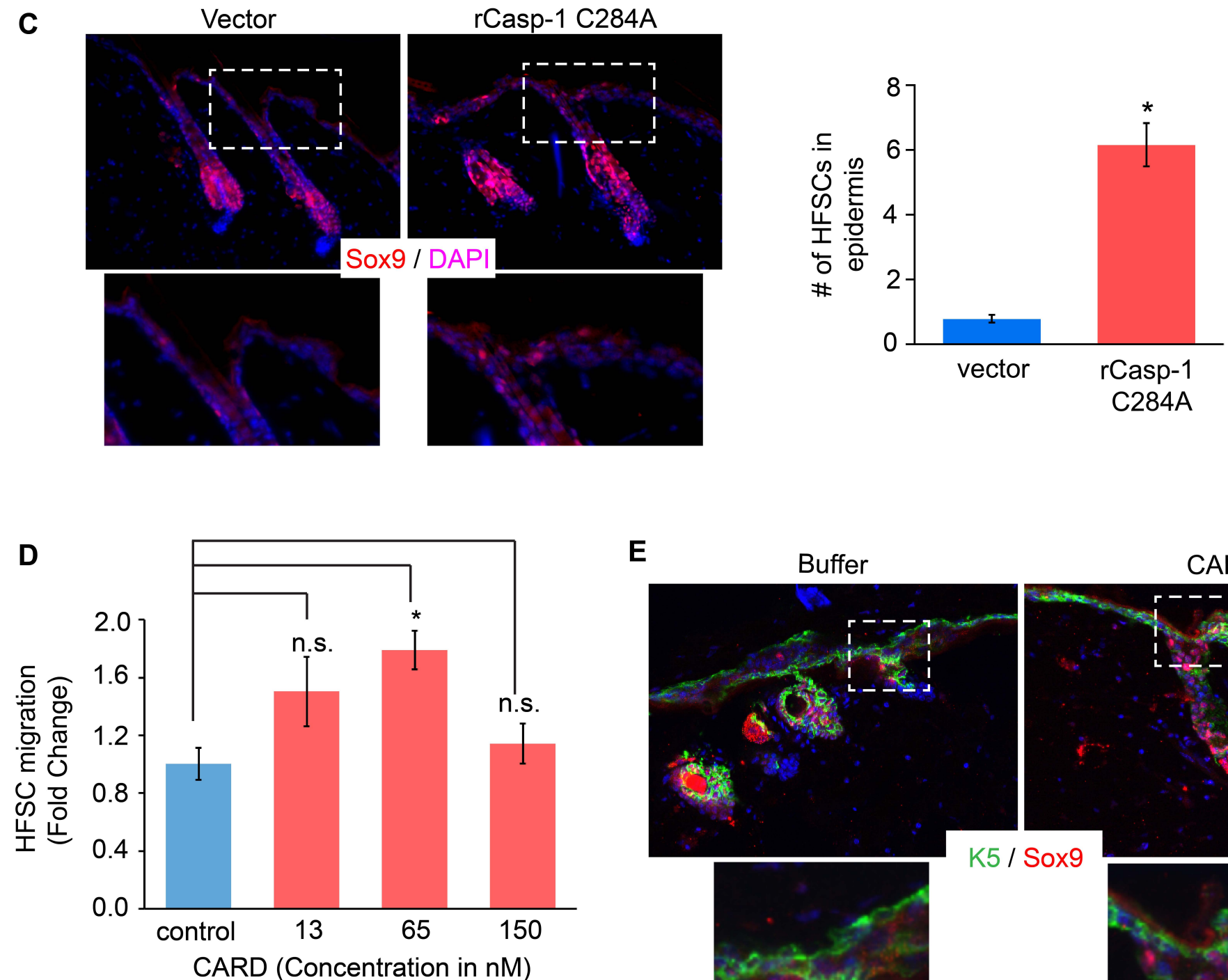

E

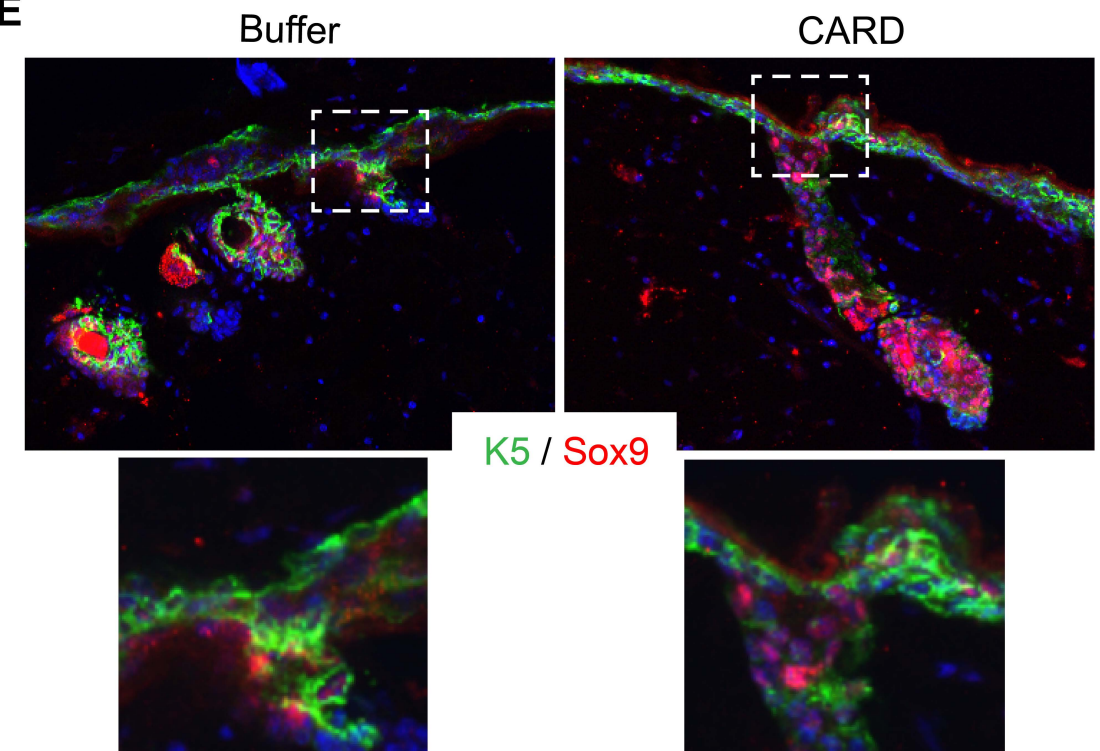


Figure 5

A

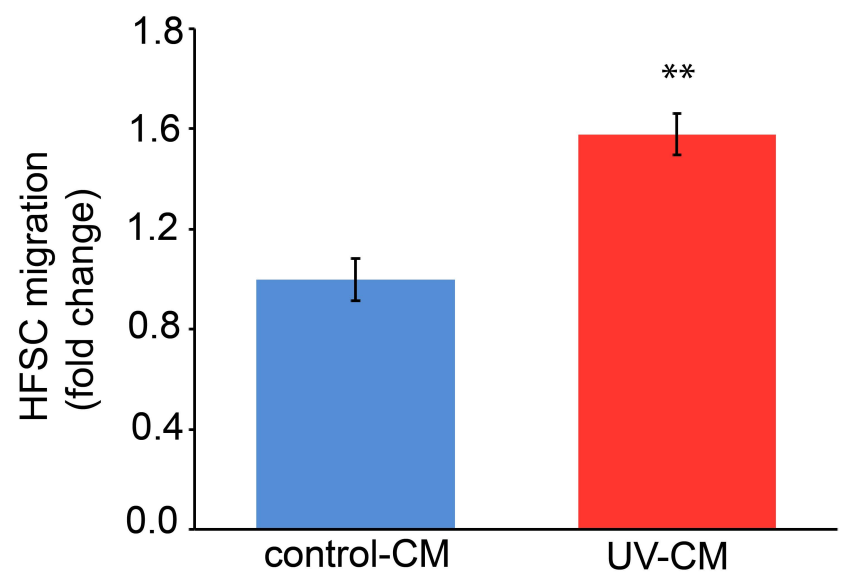

B

control

UV treated

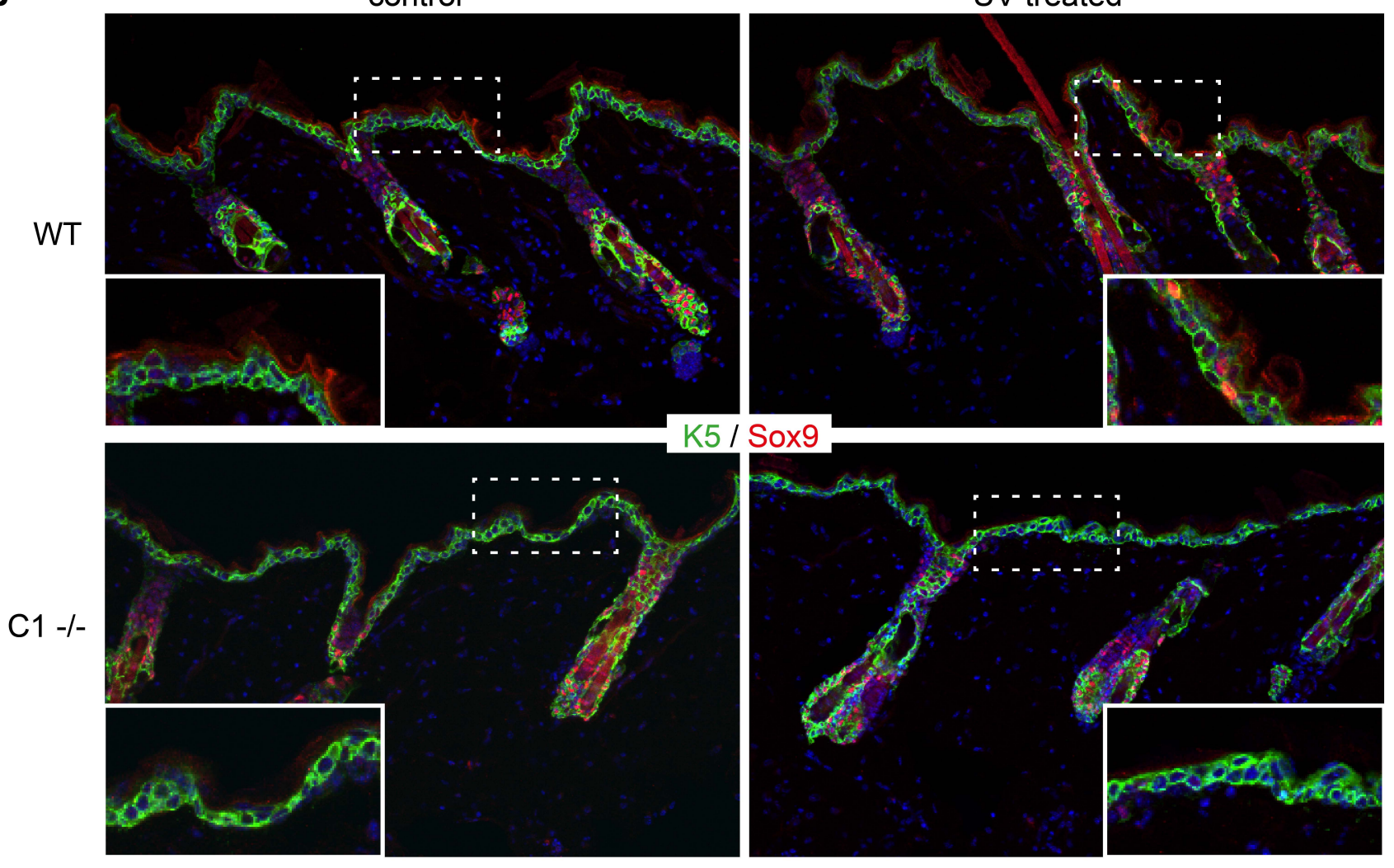

C

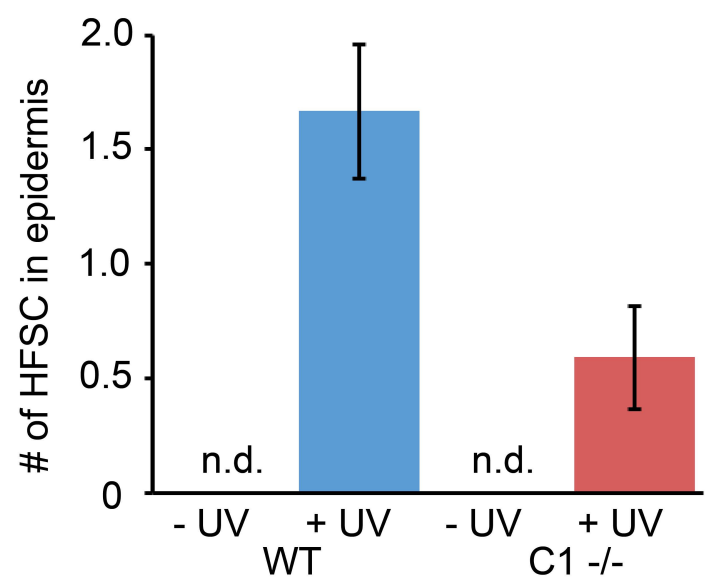

